\title{
Stereospecific Ring Contraction of Bromocycloheptenes through Dyotropic Rearrangements via Nonclassical Carbocation-Anion Pairs
}

Shermin S. Goh, ${ }^{\dagger, \|, \#}$ Pier Alexandre Champagne, ${ }^{\neq, \# \odot ~ S u r e s h b a b u ~ G u d u g u n t l a, ~}{ }^{\dagger}$ Takashi Kikuchi, ${ }^{\S, \perp}$

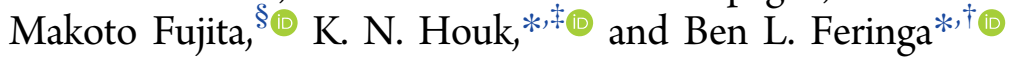

${ }^{\dagger}$ Stratingh Institute for Chemistry, University of Groningen, Nijenborgh 4, 9747 AG Groningen, The Netherlands

${ }^{*}$ Department of Chemistry and Biochemistry, University of California-Los Angeles, 607 Charles E. Young Drive East, Box 951569, Los Angeles, California 90095-1569, United States

${ }^{\S}$ Department of Applied Chemistry, University of Tokyo, 7-3-1 Hongo, Bukyo-ku, Tokyo 113-8656, Japan

${ }^{\perp}$ Rigaku Corporation, 3-9-12 Matsubara-cho, Akishima-shi, Tokyo 196-8628, Japan

\section{Supporting Information}

ABSTRACT: Experimental and theoretical evidence is reported for a rare type I dyotropic rearrangement involving a $[1,2]$-alkene shift, leading to the regio- and stereospecific ring contraction of bromocycloheptenes. This reaction occurs under mild conditions, with or without a Lewis acid catalyst. DFT calculations show that the reaction proceeds through a nonclassical carbocationanion pair, which is crucial for the low activation barrier and enantiospecificity. The chiral cyclopropylcarbinyl cation may be a transition state or an intermediate, depending on the reaction conditions.

$\mathrm{T}$ the course of our investigation of the desymmetrization of meso-3,7-dibromocycloheptene 1, we discovered that the initially formed enantiomerically enriched homoallylic bromides 2 were spontaneously isomerized with retention of configuration to form chiral substituted cyclohexenes 3 (Scheme 1). ${ }^{1}$ This reaction could be accelerated by Lewis

Scheme 1. Desymmetrization of 1 by AAS and Subsequent Rearrangement of Cycloheptenes 2 to Cyclohexenes $3^{a, b}$

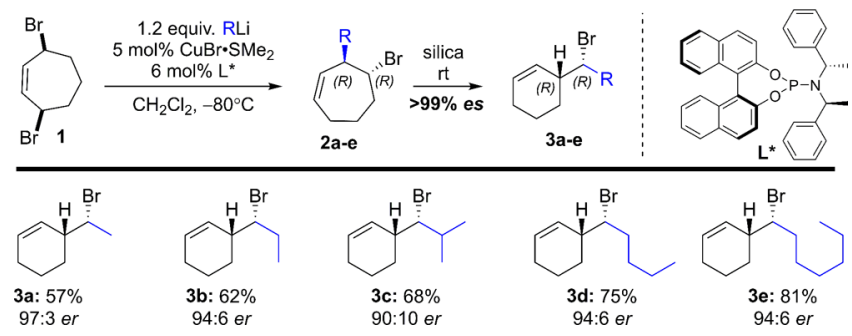

${ }^{a}$ Isolated yields over two steps. ${ }^{b}$ er's of $2 \mathbf{a}-\mathbf{e}$ and $3 \mathbf{a}-\mathbf{e}$ were determined by chiral GC to be the same, so es is $>99 \%$.

acids such as silica or $\mathrm{ZnBr}_{2}$, with no change in stereoselectivity. We have studied this reaction by a combination of experiments and computations, and now report that this involves a rare dyotropic rearrangement involving nonclassical cyclopropylcarbinyl cations on the reaction paths as either transition states or intermediates, depending on the conditions.
The copper-catalyzed desymmetrization of 1 by asymmetric allylic substitution $^{2}$ (AAS) with organolithium reagents ${ }^{3}$ initially afforded the expected products $2 \mathbf{a}-\mathbf{e}(>99: 1 d r)$, as observed by NMR spectroscopy of the crude reaction mixtures [see Supporting Information (SI)]. The reaction also proceeded with high enantioselectivity, as determined by chiral GC. ${ }^{1}$ However, upon exposure to silica, the bromocycloheptenes $\mathbf{2 a - e}$ isomerized to afford six-membered cyclic homoallylic bromides $3 \mathbf{a}-\mathbf{e}$. The ring contraction reaction proceeded with complete regioselectivity and enantiospecificity, as determined by chiral GC [see SI, part 1 and SI, part 2].

In fact, the rearrangement reaction was so facile that the transformation from cycloheptenes $\mathbf{2 a}-\mathbf{e}$ to cyclohexenes $\mathbf{3 a}-\mathbf{e}$ occurred even on neutral alumina, or when left standing in chloroform-d $\left(t_{1 / 2} \approx 6\right.$ days). The major enantiomer of $3 \mathbf{d},{ }^{4}$ obtained as a colorless oil, was determined to be $(R, R)$ by X-ray crystallography using only $5 \mu \mathrm{g}$ of compound and the crystalline sponge method developed by the Fujita group ${ }^{5}$ (Figure 1). A similar attempt was made to study the absolute

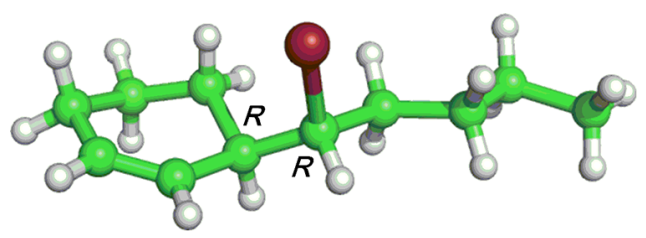

Figure 1. Ball-and-stick representation of the X-ray structure of $\mathbf{3 d}$ determined by the crystalline sponge method.

configuration of its precursor $\mathbf{2 d}$; however, the rearrangement occurred while soaking in crystalline sponge, and only $\mathbf{3 d}$ was detected when the X-ray analysis was performed.

The ring contraction reaction from $2 \mathbf{a}-\mathbf{e}$ to $3 \mathbf{a}-\mathbf{e}$, interconverting the two isomeric homoallylic bromides, involves the 1,2-positional exchange of the alkenyl and bromo groups; thus it is formally a [2,2]-dyotropic rearrangement. These were first described in 1972 by M. T. Reetz as a class of pericyclic valence isomerizations involving the simultaneous

Received: January 23, 2018

Published: March 29, 2018 
intramolecular migration of two $\sigma$-bonds. ${ }^{6,7}$ Type I dyotropic rearrangements occur when the two migrating groups exchange their positions (e.g., the classic rearrangement of anti vicinal dibromides, Scheme $2 a),{ }^{8}$ while in type II rearrangements the

Scheme 2. Uncatalyzed Concerted Type I Dyotropic

Rearrangements Occurring on a Static C-C Scaffold

(a) Vicinal dibromide dyotropic rearrangement (Ref 8)

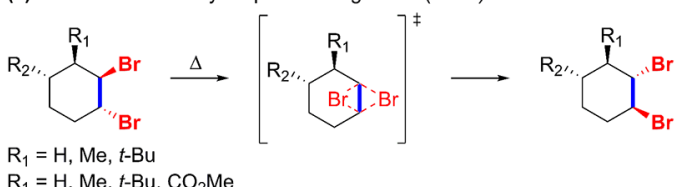

$\mathrm{R}_{1}=\mathrm{H}, \mathrm{Me}, t-\mathrm{Bu}, \mathrm{CO}_{2} \mathrm{Me}$

(b) Alkyl-mesylate dyotropic rearrangement (Ref 20)

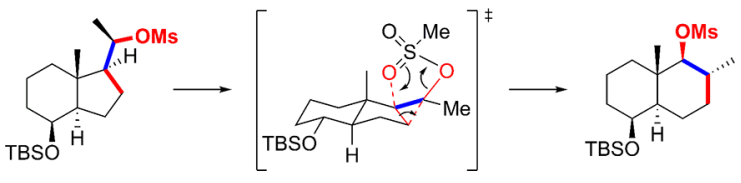

groups migrate to entirely different positions. Dyotropic reactions have become more common recently in organic and organometallic chemistry ${ }^{9}$ and have even been applied to total syntheses. 90 The concerted migration of carbon chains through a $[2,2]$-shift on a static $\mathrm{C}-\mathrm{C}$ scaffold is uncommon. $\left[\sigma^{2}+\sigma_{s} 2_{s}\right]$ processes are thermally forbidden by the Woodward-Hoffmann rules, ${ }^{11,12}$ but the reaction becomes allowed if one migrating group has a lone pair and migrates with inversion. Nevertheless, such reactions are still rare because the activation barriers are usually quite high. ${ }^{13}$

Until recently, the only experimental examples of such reactions involved highly strained lactones reacting under strenuous conditions. Examples of alkyl group migration are the ring expansion of $\beta$-lactones to butyrolactones promoted by stoichiometric magnesium bromide, ${ }^{14-16}$ or the rearrangement of cage $\delta$-lactones to $\gamma$-lactones at $350{ }^{\circ} \mathrm{C}$ on a quartz column. ${ }^{17}$ Acyl group migration involving a $\beta$-lactone ring expansion promoted by stoichiometric Lewis acid is also known. ${ }^{18}$ In recent years, milder dyotropic rearrangements have been discovered. Gutta and Tantillo proposed a 1,2-positional exchange of an alkyl group and a hydrogen atom in their computed biosynthetic pathway for formation of pentalenene in 2006. ${ }^{19}$ More recently, Faza, Lopez, and co-workers reported the type I dyotropic ring expansion of hydrindane to decalin occurring at $-78{ }^{\circ} \mathrm{C}$ upon mesylation (Scheme $2 \mathrm{~b}$ ). ${ }^{20}$ To the best of our knowledge, a concerted and uncatalyzed type I dyotropic migration of a $\mathrm{C}-\mathrm{C} \pi$ system is still unknown.

The mechanism of this rearrangement was investigated by NMR spectroscopy. Time-dependent aliquot studies with silica as reagent $(500 \% \mathrm{w} / \mathrm{w})$ for the rearrangement of bromocycloheptene $2 \mathrm{~d}$ showed that bromocyclohexene 3d (>99\% es) was the only product generated. This was observed in both polar aprotic (chloroform- $d$ ) and apolar (benzene- $d_{6}$ ) solvents, although the rate of reaction was slower in benzene- $d_{6}$ (Figure $2 \mathrm{~b}, \mathrm{c})$; the lack of solvent dependence for selectivity indicates that the reaction does not proceed through a discrete carbocation. The use of Lewis acids (e.g., $\mathrm{ZnBr}_{2}$ and TMSOTf) led to poorer selectivity in the rearrangement reaction, with ca. 5-10\% of a different diastereoisomer $4 \mathrm{~d}$ observed (Figure $2 \mathrm{~d}, \mathrm{e}){ }^{21}$ its formation may be explained by the ionization of product $\mathbf{3 d}$ by the stronger Lewis acids [see SI]. There was, fortunately, no erosion in ee of the expected product. These reactions with Lewis acids could be monitored in situ by NMR time course experiments in either chloroform- $d$ (Figure 2a) or benzene- $d_{6}$ with no difference in product ratio or enantiospecificity, although the reaction was always slower in the apolar solvent [see SI]. The reaction could also be performed with Brønsted acids [see SI], or with catalytic amounts of Lewis acids (Figure 2e), albeit at a much reduced rate. When conducted in the presence of radical scavenger BHT (1.0 equiv), the reaction profile did not change [see SI], indicating that a radical pathway was not involved.

To further understand the mechanism and specificity of the rearrangement, we performed DFT calculations on compound
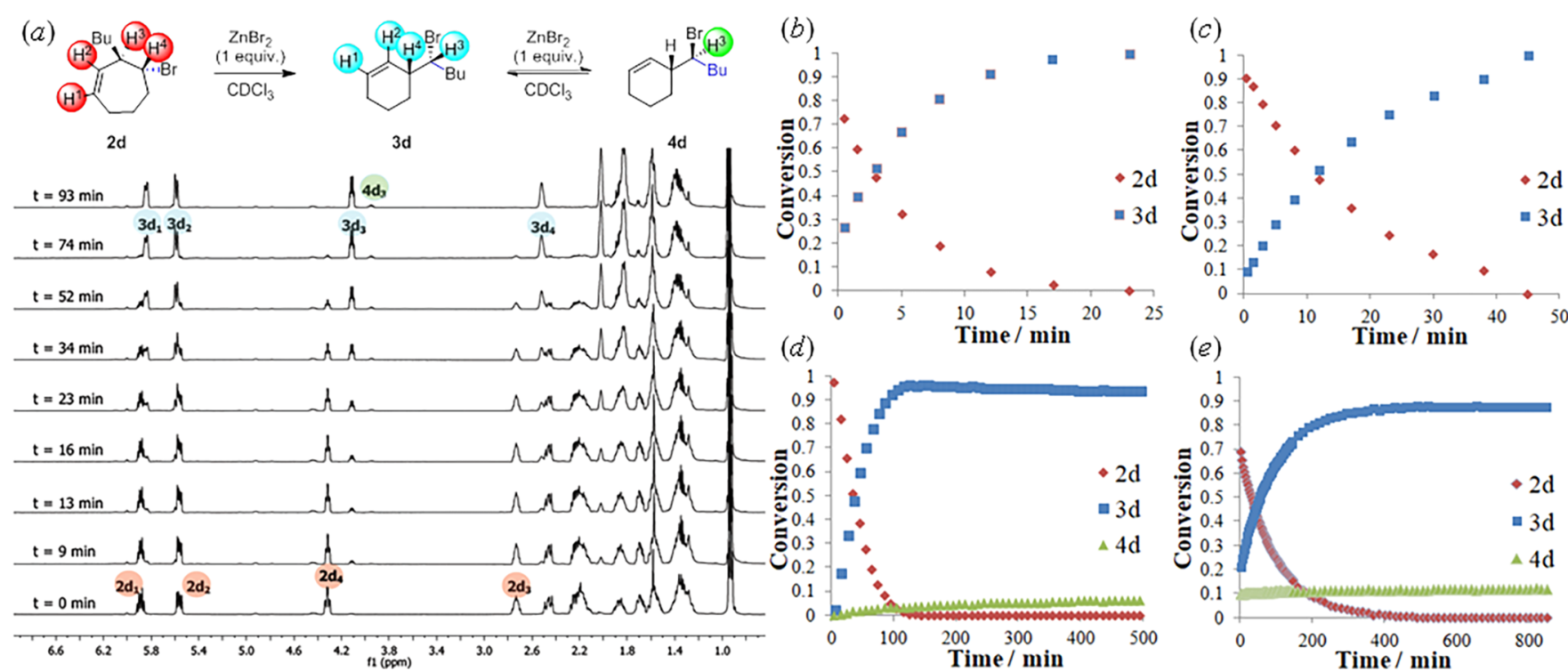

Figure 2. Left: (a) ${ }^{1} \mathrm{H}$ NMR spectroscopic profiles of bromocycloheptene $2 \mathbf{d}$ with $\mathrm{ZnBr}_{2}$ (1 equiv) in chloroform- $d$ with increasing time; reaction scheme is shown at top. Right: Reaction progress of $\mathbf{2 d}$ with silica $(500 \% \mathrm{w} / \mathrm{w})$ monitored by timed aliquots in $(\mathrm{b})$ chloroform- $d$ and $(\mathrm{c})$ benzene- $d_{6}$. Reaction progress of $\mathbf{2 d}$ monitored by in situ NMR spectroscopy with (d) $\mathrm{ZnBr}_{2}$ (1 equiv) in chloroform- $d$ and (e) TMSOTf (0.5 equiv) in chloroform- $d$. 
$2 \mathrm{a}$ at the M06-2X/6-311+G(d,p)//M06-2X/6-31G(d) level of theory, ${ }^{22}$ using Gaussian 09. ${ }^{23}$ The SMD solvation model $^{24}$ for $\mathrm{CHCl}_{3}$ was used throughout. Computed structures were visualized using CYLview. ${ }^{25}$ We first investigated whether a concerted uncatalyzed dyotropic rearrangement is plausible. The cycloheptene ring of 2a has two low-energy conformations, where the methyl and bromo substituents are either pseudoaxial (2a-ax) or pseudoequatorial (2a-eq). From 2aeq, a dyotropic rearrangement involves the experimentally observed 1,2-shift of both the alkenyl and bromo groups to form 3a. The barrier for this transformation is only $25.2 \mathrm{kcal} /$ mol (Figure 3), and the reaction is exergonic by $5.0 \mathrm{kcal} / \mathrm{mol}$.

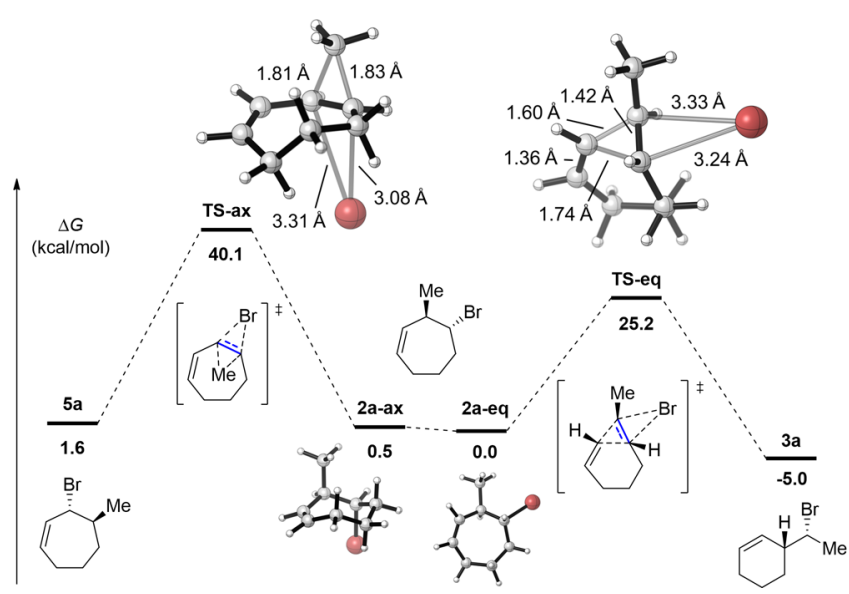

Figure 3. Calculated free energy profile for the concerted dyotropic rearrangements from the pseudoaxial (left part) or pseudoequatorial (right part) conformations of $\mathbf{2 a}$.

In contrast, the dyotropic rearrangement of the methyl and bromo groups from $\mathbf{2 a - a x}$, forming $\mathbf{5 a}$, has a significantly higher barrier of $40.1 \mathrm{kcal} / \mathrm{mol}$. The calculated $\Delta G^{\ddagger}$ for TS-eq is consistent with our observations of a slow uncatalyzed transformation of $\mathbf{2 a - e}$ to $3 \mathrm{a}-\mathbf{e}$ at room temperature in chloroform $\left(t_{1 / 2} \approx 6\right.$ days $)$.

Both TS-eq and TS-ax are highly polarized, with two long $\mathrm{C}-\mathrm{Br}$ bonds $(>3.0 \AA)$ and two short $\mathrm{C}-\mathrm{C}$ bonds, and as such can be described as tight ion-pairs of a carbocation and bromide anion. Indeed, the bromine atom in these two TSs bears an almost full negative charge ( -0.95 to $-0.97 \mathrm{au}$, see SI). This is in stark contrast to classical type I dyotropic rearrangements of vicinal dibromides, where the $\mathrm{C}-\mathrm{Br}$ bonds were calculated to be around $2.5-2.8 \AA{ }^{8 f}$ It is known that delocalization of the formed $\pi$ bond in the TSs of dyotropic rearrangements provides key stabilization. ${ }^{8 \mathrm{~d}, \mathrm{e}}$ In the case of TS-eq, the large polarization causes the carbon backbone of the substrate to approach the geometry of a $\pi \sigma$-delocalized bisected cyclopropylcarbinyl (nonclassical) cation (vide infra). ${ }^{26,27}$ This greatly stabilizes the $\pi$ bond of the TS (in blue, Figure 3) and explains why TS-eq has such a low barrier compared to TSax, for which the carbon backbone has the geometry of a lessstabilized corner-protonated cyclopropane. ${ }^{28}$

Having established that the enantiospecific uncatalyzed reaction is very likely to operate through a concerted dyotropic rearrangement, we investigated the role of an external Lewis acid (Figure 4). Formation of $\mathbf{2} \mathbf{a} \cdot \mathbf{Z n B r}_{\mathbf{2}}$ from the isolated reactants is favorable by $8.9 \mathrm{kcal} / \mathrm{mol}$ [see SI]. From this coordinated species, no concerted dyotropic transition states could be located; instead a stepwise mechanism is found.

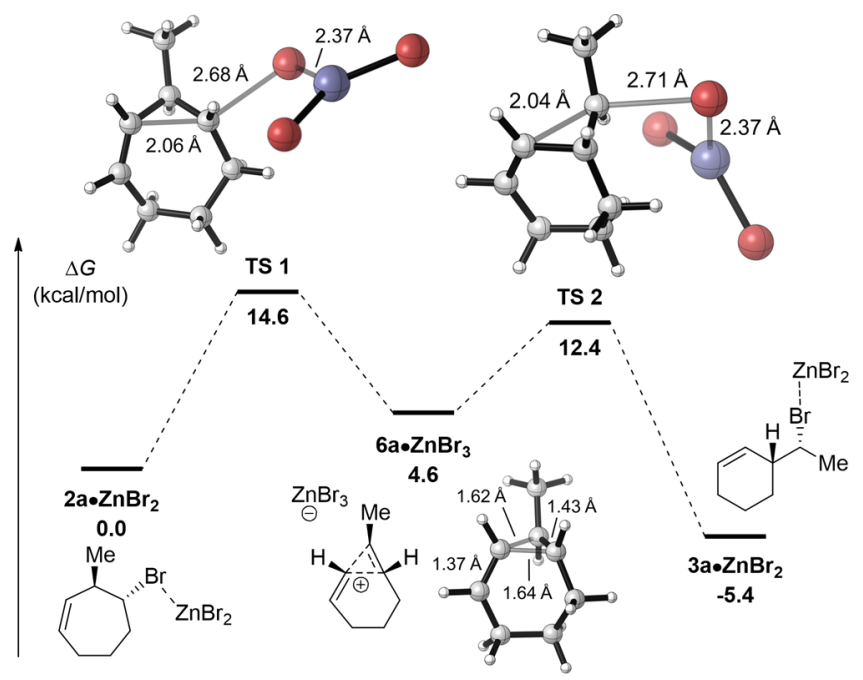

Figure 4. Calculated free energy profile for the stepwise transformation of $\mathbf{2 a}$ to $3 \mathrm{a}$, catalyzed by $\mathrm{ZnBr}_{2}$. For the structure of $\mathbf{6 a} \cdot$ $\mathrm{ZnBr}_{3}$, the $\mathrm{ZnBr}_{3}{ }^{-}$is hidden for clarity.

Ionization of the $\mathrm{C}-\mathrm{Br}$ bond of complex $2 \mathrm{a} \cdot \mathrm{ZnBr}_{2}$ through $\mathrm{TS}$ 1 has a low barrier of $14.6 \mathrm{kcal} / \mathrm{mol}$ and leads to a contact ionpair consisting of nonclassical cyclopropylcarbinyl cation $\mathbf{6 a}$ and $\mathrm{ZnBr}_{3}{ }^{-}$. From this intermediate, only $7.8 \mathrm{kcal} / \mathrm{mol}$ is required to reach TS 2, where the $\mathrm{C}-\mathrm{Br}$ bond of $3 \mathrm{a}$ is formed. These calculations are consistent with the much faster reaction observed when $\mathrm{ZnBr}_{2}$ is used as Lewis acid, since the activation barrier is predicted to be almost $10 \mathrm{kcal} / \mathrm{mol}$ lower than in the uncatalyzed case $(14.6$ vs $25.2 \mathrm{kcal} / \mathrm{mol})$. While some stepwise ionic dyotropic rearrangements are known, these were limited to intramolecular examples (e.g., dyotropic rearrangement of Himbert cycloadducts via a zwitterion). ${ }^{29,30}$ The present mechanism is an unprecedented case of a stepwise formal dyotropic rearrangement proceeding through an ion-pair.

Ionization of the $\mathrm{C}-\mathrm{Br}$ bond in TS 1 happens with simultaneous backside attack of the alkenyl group, analogous to an $\mathrm{S}_{\mathrm{N}} 2$ mechanism. Similarly, bromide attack in TS 2 occurs with release of the alkenyl group; therefore the $\mathrm{C}-\mathrm{Br}$ bonds are broken and formed from the same face of $6 \mathrm{a}$, the structure of which is determined by the stereochemistry of $\mathbf{2 a}$. Thus $\mathbf{6 a}$ is a chiral carbocation; however, even in the presence of Lewis acids such as $\mathrm{ZnBr}_{2}$, the reaction of $\mathbf{2 d}$ to $\mathbf{3 d}$ was shown to be perfectly enantiospecific. We performed additional calculations to investigate the possible mechanism of racemization of 6 a. It is now well established that cyclopropylcarbinyl cations ${ }^{26,27}$ are in equilibrium with the related bicyclobutonium cations, with the latter being more stable for the parent $\mathrm{C}_{4} \mathrm{H}_{7}{ }^{+31,32}$ For the bicyclic cyclopropylcarbinyl cation $6 \mathrm{a}$, the profile is much more complex. The lowest-energy path for the racemization of $6 \mathbf{a}$ is through the meso-bicyclobutonium 7a, which is a TS (instead of a minimum) on the potential energy surface of cation 6 a (Figure 5). ${ }^{33}$

Once chiral cation $\mathbf{6 a}$ is formed from the $\mathrm{C}-\mathrm{Br}$ bond cleavage of $2 \mathrm{a}$, it requires an additional $10.9 \mathrm{kcal} / \mathrm{mol}$ of free energy to racemize through $7 \mathbf{a} \cdot \mathbf{Z n B r}_{3}$, a bicyclobutonium ionpair. In contrast, it only needs $7.8 \mathrm{kcal} / \mathrm{mol}$ to recombine with the $\mathrm{ZnBr}_{3}{ }^{-}$counteranion to form the stable product 3a through TS 2. Moreover, the latter reaction is not reversible, as $3 a^{\cdot}$ $\mathrm{ZnBr}_{2}$ is $5.4 \mathrm{kcal} / \mathrm{mol}$ more stable than the starting complex. As such, there is a kinetic barrier to racemization in this system, allowing a stepwise enantiospecific rearrangement to occur. 

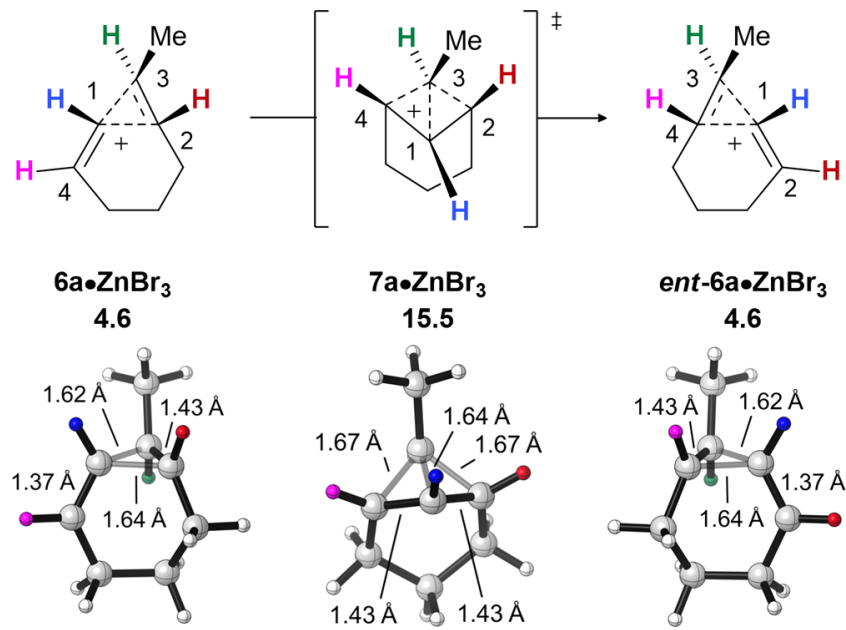

Figure 5. Lowest-energy pathway for racemization of $\mathbf{6 a} \cdot \mathbf{Z n B r}_{3}$. Free energies $(\mathrm{kcal} / \mathrm{mol})$ are relative to $\mathbf{2 a} \cdot \mathbf{Z n B r}_{2}$ (Figure 4). The $\mathrm{ZnBr}_{3}{ }^{-}$ anions are hidden for clarity.

In summary, we have discovered an unanticipated ring contraction of bromocycloheptenes under mild conditions with remarkable regio- and stereochemistry. The ring contraction occurs via a double 1,2-migration of an alkene group and a bromide. DFT calculations show that the reaction proceeds through a nonclassical carbocation-anion pair; the $\pi \sigma$ delocalized bisected cyclopropylcarbinyl cation is crucial for the low activation barrier and enantiospecificity of the rearrangement. The reaction is concerted when uncatalyzed, presenting a rare type I dyotropic rearrangement of an alkene on a $\mathrm{C}-\mathrm{C}$ stationary scaffold. In contrast, the reaction follows a stepwise mechanism under Lewis acid catalysis, and can be described as a formal dyotropic rearrangement. Our study also highlights how cations derived from chiral homoallylic halides may be productive intermediates in other enantiospecific reactions.

\section{ASSOCIATED CONTENT}

\section{S Supporting Information}

The Supporting Information is available free of charge on the ACS Publications website at DOI: 10.1021/jacs.8b00821.

Experimental procedures and characterization data, and data for computed structures (PDF)

NMR and chiral GC spectra (PDF)

X-ray crystallographic data for compound $3 \mathrm{~d}$ (CIF)

\section{AUTHOR INFORMATION}

\section{Corresponding Authors}

*houk@chem.ucla.edu

*b.l.feringa@rug.nl

\section{ORCID $\odot$}

Pier Alexandre Champagne: 0000-0002-0546-7537

Makoto Fujita: 0000-0001-6105-7340

K. N. Houk: 0000-0002-8387-5261

Ben L. Feringa: 0000-0003-0588-8435

\section{Present Address}

"S.S.G.: Institute of Materials Research and Engineering, 2 Fusionopolis Way, Innovis \#08-03, Singapore 138634

Author Contributions

${ }^{\#}$ S.S.G. and P.A.C. contributed equally.

\section{Notes}

The authors declare no competing financial interest.

\section{ACKNOWLEDGMENTS}

B.L.F. acknowledges The Netherlands Organization for Scientific Research (NWO-CW), the Royal Netherland Academy of Arts and Sciences (KNAW), and the Ministry of Education Culture and Science (Gravitation program 024.601035) for funding. S.S.G. acknowledges A*STAR (NSS) for a postdoctoral fellowship. P.A.C. acknowledges the FRQNT for a postdoctoral fellowship. We are grateful to the NSF (CHE-1361104 to K.N.H.) for financial support of this research. Computations were performed on the Hoffman2 cluster at UCLA. M.F. and T.K. acknowledge JST-ACCEL project in which M.F. is a principal investigator.

\section{REFERENCES}

(1) Desymmetrization of meso-dibromocycloalkenes with organolithium reagents: unpublished manuscript.

(2) For reviews of Cu-catalyzed AAS, see: (a) Geurts, K.; Fletcher, S. P.; van Zijl, A. W.; Minnaard, A. J.; Feringa, B. L. Pure Appl. Chem. 2008, 80, 1025-1037. (b) Harutyunyan, S. R.; den Hartog, T.; Geurts, K.; Minnaard, A. J.; Feringa, B. L. Chem. Rev. 2008, 108, 2824-2852. (c) Alexakis, A.; Bäckvall, J. E.; Krause, N.; Pàmies, O.; Diéguez, M. Chem. Rev. 2008, 108, 2796. (d) Langlois, J.-B.; Alexakis, A. In Transition Metal Catalyzed Allylic Substitution in Organic Synthesis; Kazmaier, U., Ed.; Springer-Verlag: Berlin, 2012; pp 235-268. (e) Hornillos, V.; Gualtierotti, J.-B.; Feringa, B. L. Top. Organomet. Chem. 2016, 58, 1-39. (f) Baslé, O.; Denicourt-Nowicki, A.; Crévisy, C.; Mauduit, M. In Copper-Catalyzed Asymmetric Synthesis; Alexakis, A., Krause, N., Woodward, S., Eds.; Wiley-VCH: Weinheim, 2014; pp $85-126$.

(3) For selected examples of asymmetric allylic substitution with organolithium reagents, see: (a) Pérez, M.; Fañanás-Mastral, M.; Bos, P. H.; Rudolph, A.; Harutyunyan, S. R.; Feringa, B. L. Nat. Chem. 2011, 3, 377. (b) Fañanás-Mastral, M.; Pérez, M.; Bos, P. H.; Rudolph, A.; Harutyunyan, S. R.; Feringa, B. L. Angew. Chem., Int. Ed. 2012, 51, 1922. (c) Guduguntla, S.; Gualtierotti, J.-B.; Goh, S. S.; Feringa, B. L. ACS Catal. 2016, 6, 6591. (d) Hornillos, V.; Guduguntla, S.; FañanásMastral, M.; Pérez, M.; Bos, P. H.; Rudolph, A.; Harutyunyan, S. R.; Feringa, B. L. Nat. Protoc. 2017, 12, 493.

(4) CCDC 1571256 contains the supplementary crystallographic data for this paper. These data can be obtained free of charge from The Cambridge Crystallographic Data Centre.

(5) For selected examples of crystalline sponge X-ray crystallography, see: (a) Inokuma, Y.; Yoshioka, S.; Ariyoshi, J.; Arai, T.; Hitora, Y.; Takada, K.; Matsunaga, S.; Rissanen, K.; Fujita, M. Nature 2013, 495, 461. (b) Inokuma, Y.; Yoshioka, S.; Ariyoshi, J.; Arai, T.; Fujita, M. Nat. Protoc. 2014, 9, 246. (c) Hoshino, M.; Khutia, A.; Xing, H.; Inokuma, Y.; Fujita, M. IUCrJ 2016, 3, 139.

(6) Reetz, M. T. Angew. Chem., Int. Ed. Engl. 1972, 11 (2), 129.

(7) Reetz, M. T. Angew. Chem., Int. Ed. Engl. 1972, 11 (2), 130.

(8) For selected experimental and theoretical studies on the dyotropic rearrangement of anti vicinal dibromides, see: (a) Grob, C. A.; Winstein, S. Helv. Chim. Acta 1952, 35 (3), 782. (b) Barton, D. H. R.; Head, A. J. J. Chem. Soc. 1956, 932. (c) Barili, P. L.; Bellucci, G.; Berti, G.; Marioni, F.; Marsili, A.; Morelli, I. J. Chem. Soc. D 1970, 1437. (d) Frontera, A.; Suner, G. A.; Deya, P. M. J. Org. Chem. 1992, 57 (25), 6731. (e) Zou, J.-W.; Yu, C.-H. J. Phys. Chem. A 2004, 108, 5649. (f) Fernández, I.; Sierra, M. A.; Cossío, F. P. Chem. - Eur. J. 2006, 12 (24), 6323.

(9) Fernández, I.; Cossío, F. P.; Sierra, M. A. Chem. Rev. 2009, 109 (12), 6687.

(10) For selected examples of total syntheses involving dyotropic rearrangements, see: (a) Kocienski, P.; Barber, C. Pure Appl. Chem. 1990, 62 (10), 1933. (b) Lin, S.; Danishefsky, S. J. Angew. Chem., Int. Ed. 2002, 41 (3), 512. (c) Li, W.; LaCour, T. G.; Fuchs, P. L. J. Am. 
Chem. Soc. 2002, 124, 4548. (d) Denmark, S. E.; Montgomery, J. I. Angew. Chem., Int. Ed. 2005, 44 (24), 3732.

(11) Reetz, M. T. Tetrahedron 1973, 29 (14), 2189.

(12) Reetz, M. T. Adv. Organomet. Chem. 1977, 16, 33.

(13) Gutierrez, O.; Tantillo, D. J. J. Org. Chem. 2012, 77 (20), 8845.

(14) Mulzer, J.; Brütrup, G. Angew. Chem., Int. Ed. Engl. 1979, 18 (10), 793.

(15) Black, T. H.; DuBay, W. J.; Tully, P. S. J. Org. Chem. 1988, 53 (25), 5922.

(16) Arrastia, I.; Lecea, B.; Cossío, F. P. Tetrahedron Lett. 1996, 37 (2), 245.

(17) Satō, K.; Yamashita, Y.; Mukai, T. Tetrahedron Lett. 1981, 22 (52), 5303.

(18) Leverett, C. A.; Purohit, V. C.; Johnson, A. G.; Davis, R. L.; Tantillo, D. J.; Romo, D. J. Am. Chem. Soc. 2012, 134 (32), 13348.

(19) Gutta, P.; Tantillo, D. J. J. Am. Chem. Soc. 2006, 128 (18), 6172.

(20) Santalla, H.; Faza, O. N.; Gómez, G.; Fall, Y.; Silva López, C. Org. Lett. 2017, 19 (13), 3648.

(21) More experimental data for the rearrangement of $\mathbf{2 d}$ to $3 \mathrm{~d}$ monitored by NMR spectroscopy can be found in the SI.

(22) Zhao, Y.; Truhlar, D. G. Theor. Chem. Acc. 2008, 120, 215.

(23) Frisch, M. J.; et al. Gaussian 09, Revision D.01; Gaussian, Inc.: Wallingford, CT, 2013.

(24) Marenich, A. V.; Cramer, C. J.; Truhlar, D. G. J. Phys. Chem. B 2009, 113 (18), 6378.

(25) Legault, C. Y. CYLview, 1.0b; Université de Sherbrooke, 2009; http://www.cylview.org.

(26) Roberts, J. D.; Mazur, R. H. J. Am. Chem. Soc. 1951, 73 (7), 3542.

(27) Roberts, J. D.; Mazur, R. H. J. Am. Chem. Soc. 1951, 73 (6), 2509.

(28) (a) Dewar, M. J. S.; Healy, E. F.; Ruiz, J. M. J. Chem. Soc., Chem. Commun. 1987, 943. (b) Burritt, A.; Coxon, J. M.; Steel, P. J. J. Org. Chem. 1995, 60 (23), 7670.

(29) Pham, H. V.; Karns, A. S.; Vanderwal, C. D.; Houk, K. N. J. Am. Chem. Soc. 2015, 137 (21), 6956.

(30) Davis, R. L.; Leverett, C. A.; Romo, D.; Tantillo, D. J. J. Org. Chem. 2011, 76 (17), 7167.

(31) Staral, J. S.; Yavari, I.; Roberts, J. D.; Prakash, G. K. S.; Donovan, D. J.; Olah, G. A. J. Am. Chem. Soc. 1978, 100 (25), 8016.

(32) Olah, G. A.; Surya Prakash, G. K.; Rasul, G. J. Am. Chem. Soc. 2008, 130 (28), 9168.

(33) The full free energy profile of $\mathbf{6 a}$ can be found in the SI. 